\title{
Erratum: Poincaré constraints on celestial amplitudes
}

\section{Y.T. Albert Law and Michael Zlotnikov}

Department of Physics, Center for Theoretical Physics, Columbia University, 538 West 120th Street, New York, NY 10027, U.S.A.

E-mail: yal2109@columbia.edu, mz2737@columbia.edu

ERRATUM TO: JHEP03(2020)085

ABSTRACT: We provide corrections to equations (5.31), (5.32) and (5.33) in the original manuscript. The corrections remove typos due to which some terms in the results were omitted. The concepts, derivation strategy and conclusions remain unchanged.

ARXIV EPRINT: 1910.04356

\section{Section 5 corrections}

Equations (5.31), (5.32) and (5.33) in the original manuscript [1] are to be replaced by

$$
\begin{aligned}
& 0=\frac{m_{4} \epsilon_{4} \sqrt{z \bar{z}}}{\Delta_{4}-1}\left(\left(\frac{\Delta_{4}-1}{2}+\left(h_{2}-h_{1}\right) z+h_{3}\right) f_{\bar{h}_{1}, \bar{h}_{2}, \bar{h}_{3}, \frac{\Delta_{4}-1}{2}}^{h_{1}, h_{2}, h_{3}, \frac{\Delta_{4}-1}{2}}+(z-1) z \partial_{z} f_{\bar{h}_{1}, \bar{h}_{2}, \bar{h}_{3}, \frac{\Delta_{4}-1}{2}}^{h_{1}, h_{2}, h_{3}, \frac{\Delta_{4}-1}{2}}\right)
\end{aligned}
$$

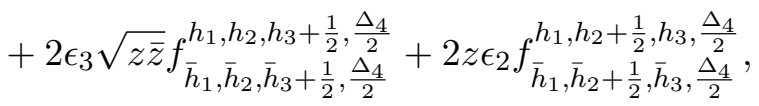

$$
\begin{aligned}
& 0=\frac{m_{4} \epsilon_{4} \sqrt{z \bar{z}}}{\Delta_{4}-1}\left(\left(\frac{\Delta_{4}-1}{2}+\left(\bar{h}_{2}-\bar{h}_{1}\right) \bar{z}+\bar{h}_{3}\right) f_{\bar{h}_{1}, \bar{h}_{2}, \bar{h}_{3}, \frac{\Delta_{4}-1}{2}}^{h_{1}, h_{2}, h_{3}, \frac{\Delta_{4}-1}{2}}+(\bar{z}-1) \bar{z} \partial_{\bar{z}} f_{\bar{h}_{1}, \bar{h}_{2}, \bar{h}_{3}, \frac{\Delta_{4}-1}{2}}^{h_{1}, h_{2}, h_{3}, \frac{\Delta_{4}-1}{2}}\right) \\
& +2 \epsilon_{3} \sqrt{z \bar{z}} f_{\bar{h}_{1}, \bar{h}_{2}, \bar{h}_{3}+\frac{1}{2}, \frac{\Delta_{4}}{2}}^{h_{1}, h_{2}, h_{3}+\frac{\Delta_{4}}{2}}+2 \bar{z} \epsilon_{2} f_{\bar{h}_{1}, \bar{h}_{2}+\frac{1}{2}, \bar{h}_{3}, \frac{\Delta_{4}}{2}}^{h_{1}, h_{2}+\frac{1}{2}, h_{3},} \\
& 0=\frac{m_{4} \epsilon_{4} \sqrt{z \bar{z}}}{\Delta_{4}-1}\left(\left(\frac{1-\Delta_{4}}{2}+\left(h_{1}-h_{2}\right) z-h_{3}\right)\left(\frac{\Delta_{4}-1}{2}+\left(\bar{h}_{2}-\bar{h}_{1}\right) \bar{z}+\bar{h}_{3}\right) f_{\bar{h}_{1}, \bar{h}_{2}, \bar{h}_{3}, \frac{\Delta_{4}-1}{2}}^{h_{1}, h_{2}, h_{3}}\right. \\
& \left.+z \bar{z}(z-1)(\bar{z}-1) \partial_{z} \partial_{\bar{z}} f_{\bar{h}_{1}, \bar{h}_{2}, \bar{h}_{3}, \frac{\Delta_{4}-1}{2}}^{h_{1}, h_{2}, h_{4}-1}\right)+m_{4} \epsilon_{4} \Delta_{4} \sqrt{z \bar{z}} f_{\bar{h}_{1}, \bar{h}_{2}, \bar{h}_{3}, \frac{\Delta_{4}+1}{2}}^{h_{1}, h_{2}, h_{3}} \\
& +\epsilon_{2}\left(2\left(\Delta_{1}-\Delta_{2}+\Delta_{4}-1\right) z \bar{z}-z\left(2 \bar{h}_{3}+\Delta_{4}-1\right)-\bar{z}\left(\Delta_{4}+2 h_{3}-1\right)\right) f_{\bar{h}_{1}, \bar{h}_{2}+\frac{1}{2}, \bar{h}_{3}, \frac{\Delta_{4}}{2}}^{h_{1}, h_{2}+\frac{1}{2}, h_{3}, \frac{\Delta_{4}}{2}} \\
& +2 \epsilon_{3} \sqrt{z \bar{z}}\left(\left(\bar{h}_{1}-\bar{h}_{2}\right) \bar{z}+\left(h_{1}-h_{2}\right) z-\Delta_{3}\right) f_{\bar{h}_{1}, \bar{h}_{2}, \bar{h}_{3}+\frac{1}{2}, \frac{\Delta_{4}}{h_{1}, h_{2}, h_{2}},}^{\frac{1}{2}, \frac{\Delta_{4}}{2}},
\end{aligned}
$$

respectively. These corrections remove typos due to which some terms in the results were omitted. All concepts, derivation strategy and conclusions remain unchanged. 
Open Access. This article is distributed under the terms of the Creative Commons Attribution License (CC-BY 4.0), which permits any use, distribution and reproduction in any medium, provided the original author(s) and source are credited.

\section{References}

[1] Y.T.A. Law and M. Zlotnikov, Poincaré constraints on celestial amplitudes, JHEP 03 (2020) 085 [arXiv: 1910.04356] [INSPIRE]. 